\title{
Briófitas de área sob o domínio fitogeográfico do Cerrado e novas ocorrências para o Maranhão e região Nordeste do Brasil
}

\author{
Regigláucia Rodrigues de Oliveira', Dailma Lima Medeiros², Hermeson Cassiano de Oliveira ${ }^{3}$ \\ \& Gonçalo Mendes da Conceição'
}

\begin{abstract}
' Universidade Estadual do Maranhão, Programa de Pós-Graduação em Biodiversidade, Ambiente e Saúde, Praça Duque de Caxias, S/N, Morro do Alecrim, CEP 65.604-380, Caxias, Maranhão. regiglaucia@hotmail.com, doutorgoncalo@gmail.com ${ }^{2}$ Universidade Estadual do Maranhão, Laboratório de Biologia Vegetal, Praça Duque de Caxias, s/n, Morro do Alecrim, CEP 65.604-380, Caxias, Maranhão. dailmamedeiros@hotmail.com ${ }^{3}$ Universidade Estadual do Piaú, Programa de Pós-Graduação em Biodiversidade, Ambiente e Saúde. Avenida Santo Antônio, S/N, São Luís, CEP 64.280-000 Campo Maior, Piauí. hermeson123@gmail.com
\end{abstract}

RESUMO- O levantamento de briófitas realizado no município de Caxias, Maranhão resultou em 34 espécies, sendo 24 pertencentes à divisão Bryophyta, distribuídas em 16 gêneros e 11 famílias, e 10 espécies pertencentes à Marchantiophyta, distribuídas em oito gêneros e cinco famílias. Das 34 espécies identificadas Barbula arcuata Griff. configura-se como novos registro para a região Nordeste do Brasil e oito são novas ocorrências para o Maranhão. Estes resultados vem enriquecer o conhecimento brioflorístico acerca das espécies de briófitas que ocorrem no Maranhão e região Nordeste do Brasil.

Palavras-chave: Bryophyta, hepáticas, Marchantiophyta, musgos

ABSTRACT- Bryophytes of an area under the phytogeographical domain Cerrado and new records for Maranhão and the Northeast (Brazil). The study of bryophytes collected in the municipality of Caxias, Maranhão, resulted in 34 species. Twenty-four were of the Division Bryophyta, distributed in 16 genera and 11 families, and 10 species belonging to Marchantiophyta, distributed in eight genera and five families. Of the 34 species identified, Barbula arcuata Griff. is as new record for northeastern Brazil and eight were new occurrences for Maranhão. These results increase the bryofloristic knowledge in Maranhão and northeastern Brazil.

Keywords: Bryophyta, liverworts, Marchantiophyta, mosses

\section{INTRODUÇÃO}

Briófitas é o termo utilizado para designar um grupo de plantas terrestres em sua maioria, normalmente pequenas (a maioria até $10 \mathrm{~cm}$ de comprimento), criptógamas, poiquilohídrícas e ciclo de vida marcado pela alternância de gerações (Proctor et al. 2007, Goffinet et al. 2009, Glime 2013). Formam o segundo maior grupo de vegetais, sendo superada apenas pelas Angiospermas, no que diz respeito ao número de espécies (Buck \& Goffinet 2000, Goffinet \& Shaw 2009). São representadas por três diferentes grupos vegetais, os musgos, as hepáticas e os antóceros; que compõem as divisões ou filos Bryophyta (Goffinet \& Shaw 2009), Marchantiophyta (CrandallStotler et al. 2009) e Anthocerotophyta (Renzaglia et al. 2009), respectivamente.

Possuem ampla distribuição, aproximadamente 15.000 espécies conhecidas para o mundo (Gradstein et al. 2001). Para o Brasil são conhecidas cerca de 117 famílias, 413 gêneros e 1.524 espécies de briófitas, destas 149 são endêmicas. No país, atualmente, ocorrem 11 espécies de antóceros; 633 de hepáticas e 880 de musgos (Costa \& Peralta 2015).

No Nordeste brasileiro, as briófitas distribuem-se em 79 famílias, 228 gêneros e 689 espécies (Costa \& Peralta 2015). Yano et al. (2009) registraram 143 táxons de briófitas que foram coletadas por vários coletores nos estados da região Nordeste, tais como: Ceará, Paraíba, Piauí, Rio Grande do Norte e Maranhão, que estão depositadas no Herbário Científico do Estado "Maria Eneyda P. Kauffmann Fidalgo", do Instituto de Botânica (SP). Dessas, uma espécie pertence à Anthocerotophyta e os demais estão distribuídos em 81 espécies de Bryophyta em 43 gêneros e 24 famílias, e 61 de Marchantiophyta, em 33 gêneros e 13 famílias.

Pesquisas com briófitas no estado do Maranhão têm aumentado nos últimos anos, como mostra os resultados de Peralta et al. (2011) onde foram registradas 137 espécies de briófitas distribuídas em 32 famílias e 76 gêneros para o estado do Maranhão, nesse trabalho foram destacadas 
as espécies caracterizadas como ocorrências novas para o Estado (65 espécies, ou seja 47\% de novidades para o estado). Segundo Costa \& Peralta (2015), o Maranhão possui 89 espécies pertencentes a esse grupo vegetal. Porém, mais recentemente, Vieira et al. (2017) registraram 28 espécies de briófitas, distribuídas em 18 gêneros e 12 famílias. Com esse trabalho houve a adição de três espécies para o estado do Maranhão, elevando o número de briófitas desse Estado para 92 espécies.

Desta forma, o estudo objetiva enriquecer e ampliar o conhecimento das espécies da brioflora da região Nordeste do Brasil, e do Maranhão, uma vez que o Estado localizase em uma região de transição entre as fitofisionomias de Cerrado, Caatinga e com influências de ecossistemas amazônicos.

\section{MATERIAL E MÉTODOS}

A pesquisa foi realizada no município de Caxias $\left(04^{\circ} 53^{\prime} 30\right.$ "S e $43^{\circ} 24^{\prime} 53^{\prime}$ 'W), com coletas feitas no povoado Buriti Corrente ( $4^{\circ} 45^{\prime} 17,62^{\prime}$ " S e $43^{\circ} 39^{\prime} 31$, 62 "O). A área situa-se às margens da BR-316, distante ca. de $37 \mathrm{~km}$ do perímetro urbano de Caxias, banhado pelo riacho Prata. A vegetação é típica do domínio fitogeográfico do Cerrado, com presença de floresta de galeria margeando o riacho, manchas de pastagens e florestas secundárias com grande predominância de babaçuais e buritizais.

As amostras foram coletadas no período de janeiro a maio de 2015, em trilhas com caminhadas aleatórias e no interior da mata, percorrendo a maior extensão possível do riacho Prata, em todos os substratos disponíveis que as briófitas pudessem colonizar, tais como: rocha, solo, troncos de árvore vivos, troco de árvores em decomposição entre outros. Foram observados e anotados os substratos de cada espécime coletado.

Os processos de coleta, armazenamento e herborização basearam-se em Gradstein et al. (2001). As espécies foram identificadas utilizando-se os trabalhos de Schuster (1980), Zander (1993), Sharp et al. (1994), Buck (2003) e Gradstein $\&$ Costa (2003), com a confirmação e/ou identificação de especialista no grupo em estudo.

A distribuição geográfica baseou-se em dados disponíveis na plataforma Flora do Brasil (2020), e trabalhos recentemente publicados como: Vieira et al. (2017) para o estado do Maranhão e Batista et al (2018) para a região Nordeste. As amostras foram devidamente identificadas e incorporadas à Coleção Briológica do Herbário Prof. Aluízio Bittencourt (HABIT). Ressaltase que os vouchers foram incluídos na Lista de espécies (Quadro 1).
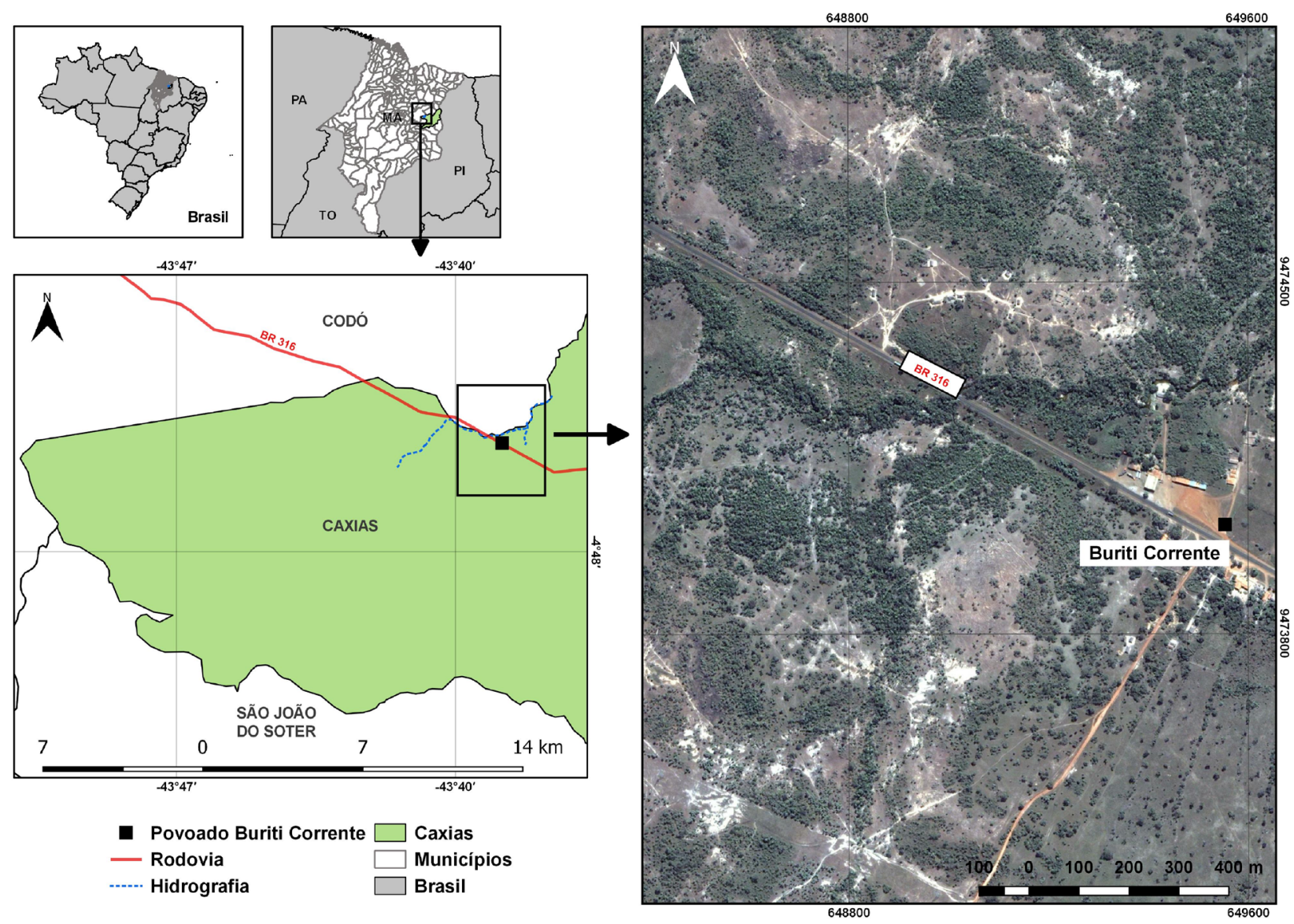

Fig. 1. Mapa com a localização da área de coleta do povoado Buriti Corrente, município de Caxias, Maranhão, Brasil. 
Quadro 1. Lista de espécies com os respectivos substratos de ocorrência $($ Cas $=$ Casmófita, Co $=$ Corticícola, Cup $=$ Cupinzeiro, Ep $=$ Epixílica, Rup $=$ Rupícola, Ter = Terrícola), com a distribuição geográfica no Brasil. *Nova ocorrência para o estado do Maranhão ** Nova ocorrência para a região Nordeste do Brasil.

\begin{tabular}{|c|c|c|c|}
\hline Divisão/ Famílias / Espécies & Substratos & Distribuição Geográfica no Brasil & Voucher \\
\hline \multicolumn{4}{|l|}{ Bryophyta } \\
\hline \multicolumn{4}{|l|}{ Archidiaceae } \\
\hline Archidium ohioense Schimp. ex Müll. Hal. & Ter & $\begin{array}{c}\mathrm{AL}, \mathrm{BA}, \mathrm{CE}, \mathrm{PB}, \mathrm{PE}, \mathrm{PI}, \mathrm{RN}, \mathrm{GO}, \mathrm{MS}, \mathrm{MT}, \\
\mathrm{MG}, \mathrm{RS} .\end{array}$ & $\begin{array}{l}\text { Medeiros, D.L. \& Conceição, } \\
\text { G.M. } 382 \text { (HABIT732) }\end{array}$ \\
\hline \multicolumn{4}{|l|}{ Bartramiaceae } \\
\hline Philonotis sphaerocarpa (Hedw.) Brid. & Ter & AM, BA, CE, MT, RJ, SP, SC. & $\begin{array}{l}\text { Medeiros, D.L. \& Conceição, } \\
\text { G.M.360 (HABIT716) }\end{array}$ \\
\hline \multicolumn{4}{|l|}{ Bryaceae } \\
\hline Bryum apiculatum Schwägr. & Ter e Ep & $\begin{array}{c}\mathrm{AC}, \mathrm{PA}, \mathrm{CE}, \mathrm{DF}, \mathrm{GO}, \mathrm{MS}, \mathrm{MG}, \mathrm{RJ}, \mathrm{SP}, \mathrm{PR}, \\
\text { SC. }\end{array}$ & $\begin{array}{l}\text { Medeiros, D.L. \& Conceição, } \\
\text { G.M.384 (HABIT722) }\end{array}$ \\
\hline \multicolumn{4}{|l|}{ Calymperaceae } \\
\hline Calymperes palisotii Schwägr. & Ter e Co & $\begin{array}{c}\text { AM, AP, PA, RO, RR, TO } \\
\text { AL, BA, MA, PB, PE, PI, RN, SE, GO, MS, } \\
\text { MT, ES, MG, RJ, SP, PR. }\end{array}$ & $\begin{array}{l}\text { Medeiros, D.L. \& Conceição, } \\
\text { G.M.370 (HABIT775) }\end{array}$ \\
\hline C. pallidum Mitt. & Ter & AC, AM, PA, RO, BA, GO, MT. & $\begin{array}{l}\text { Medeiros, D.L. \& Conceição, } \\
\text { G.M.1165 (HABIT667) }\end{array}$ \\
\hline Octoblepharum albidum Hedw. & Ter, Co, Ep e Cup & $\begin{array}{l}\text { AC, AM, AP, PA, RO, RR, TO, AL, BA, CE, } \\
\text { MA, PB, PE, PI, RN, SE, DF, GO, MS, MT, } \\
\text { ES, MG, RJ, SP, PR, RS, SC. }\end{array}$ & $\begin{array}{l}\text { Medeiros, D.L. \& Conceição, } \\
\text { G.M.274 (HABIT732) }\end{array}$ \\
\hline Syrrhopodon gardneri (Hook. f.) Schwägr. & $\mathrm{Co}$ & AM, BA, GO, MT, MG, RJ. & $\begin{array}{l}\text { Medeiros, D.L. \& Conceição, } \\
\text { G.M.245 (HABIT731) }\end{array}$ \\
\hline \multicolumn{4}{|l|}{ Ditrichaceae } \\
\hline Garckea flexuosa (Griff.) Margad. \& Nork. & Ter & TO, MT. & $\begin{array}{l}\text { Medeiros, D.L. \& Conceição, } \\
\text { G.M. } 382 \text { (HABIT742) }\end{array}$ \\
\hline \multicolumn{4}{|l|}{ Fissidentaceae } \\
\hline Fissidens goyazensis Broth. & Ter, Co e Ep & $\begin{array}{l}\mathrm{AM}, \mathrm{BA}, \mathrm{CE}, \mathrm{PB}, \mathrm{PE}, \mathrm{PI}, \mathrm{DF}, \mathrm{GO}, \mathrm{MG}, \mathrm{RJ} \\
\text { SP. }\end{array}$ & $\begin{array}{l}\text { Medeiros, D.L. \& Conceição, } \\
\text { G.M.231 (HABIT732) }\end{array}$ \\
\hline F. palmatus Hedw. & Ter e Cas & $\mathrm{AC}, \mathrm{BA}, \mathrm{CE}, \mathrm{MA}, \mathrm{PE}, \mathrm{GO}, \mathrm{SP}$. & $\begin{array}{l}\text { Medeiros, D.L. \& Conceição, } \\
\text { G.M.256 (HABIT700) }\end{array}$ \\
\hline F. angustelimbatus Mitt. & Ter e Ep & $\begin{array}{c}\mathrm{AC}, \mathrm{RO}, \mathrm{RR}, \mathrm{TO}, \mathrm{BA}, \mathrm{MA}, \mathrm{DF}, \mathrm{GO}, \mathrm{MT}, \\
\text { MG, SP, PR, RS. }\end{array}$ & $\begin{array}{l}\text { Medeiros, D.L. \& Conceição, } \\
\text { G.M.271 (HABIT696) }\end{array}$ \\
\hline F. pellucidus Hornsch. & Ter & $\begin{array}{l}\text { AC, AM, PA, RO, RR, TO, BA, CE, PB, PE, } \\
\text { DF, GO, MT, ES, MG, RJ, SP, PR, RS, SC. }\end{array}$ & $\begin{array}{l}\text { Medeiros, D.L. \& Conceição, } \\
\text { G.M.382 (HABIT732) }\end{array}$ \\
\hline F. serratus Müll. Hal.* & Cup & $\begin{array}{c}\text { AM, BA, CE, PE, PI, GO, MT, ES, MG, RJ, } \\
\text { SP, RS, SC. }\end{array}$ & $\begin{array}{l}\text { Medeiros, D.L. \& Conceição, } \\
\text { G.M.225 (HABIT748) }\end{array}$ \\
\hline F. flaccidus Mitt. & Ter & $\begin{array}{l}\text { AC, AM, PA, RO, TO, BA, CE, MA, PB, } \\
\text { PE, SE, DF, GO, MS, MT, ES, MG, RJ, SP, } \\
\text { PR, RS. }\end{array}$ & $\begin{array}{l}\text { Medeiros, D.L. \& Conceição, } \\
\text { G.M.157 (HABIT838) }\end{array}$ \\
\hline \multicolumn{4}{|l|}{ Hypnaceae } \\
\hline Isopterygium tenerifolium Mitt. & Ter e Ep & $\begin{array}{c}\text { AM, PA, RO, BA, CE, DF, GO, MT, MG, RJ, } \\
\text { SP, PR, RS, SC. }\end{array}$ & $\begin{array}{l}\text { Medeiros, D.L. \& Conceição, } \\
\text { G.M.258 (HABIT688) }\end{array}$ \\
\hline Isopterygium affusum Mitt.* & Ter e Co & AM, PA, CE, GO, MT, MG, RJ, SP, SC. & $\begin{array}{l}\text { Medeiros, D.L. \& Conceição, } \\
\text { G.M. } 275 \text { (HABIT694) }\end{array}$ \\
\hline $\begin{array}{l}\text { Chryso-hypnum diminutivum (Hampe) } \\
\text { W.R. Buck }\end{array}$ & Ter & $\begin{array}{l}\text { AC, AM, AP, PA, RO, RR, TO, BA, PE, DF, } \\
\text { GO, MS, MT, ES, MG, RJ, SP, PR, RS, SC. }\end{array}$ & $\begin{array}{l}\text { Medeiros, D.L. \& Conceição, } \\
\text { G.M.343 (HABIT803) }\end{array}$ \\
\hline \multicolumn{4}{|l|}{ Pottiaceae } \\
\hline Barbula arcuata Griff.** & Ter & AM, GO, MG, RJ, SP. & $\begin{array}{c}\text { Medeiros, D.L. \& Conceição, } \\
\text { G.M.272 } \\
\text { (HABIT697) }\end{array}$ \\
\hline B. indica (Hook.) J.R. Spreng.* & Ter e Cas & $\begin{array}{c}\text { AC, AM, PA, BA, PE, RN, SE, DF, GO, MS, } \\
\text { MT, ES, MG, RJ, SP, PR, RS. }\end{array}$ & $\begin{array}{l}\text { Medeiros, D.L. \& Conceição, } \\
\text { G.M.352 (HABIT793) }\end{array}$ \\
\hline \multicolumn{4}{|l|}{ Sematophyllaceae } \\
\hline $\begin{array}{l}\text { Trichosteleum subdemissum (Schimp. ex } \\
\text { Besch.) A. Jaeger }\end{array}$ & Co, Ter, Ep & $\begin{array}{c}\text { AM, PA, RO, RR, BA, MA, PI, DF, GO, MT, } \\
\text { MG, RJ, SP. }\end{array}$ & $\begin{array}{l}\text { Medeiros, D.L. \& Conceição, } \\
\text { G.M.248 (HABIT715) }\end{array}$ \\
\hline $\begin{array}{l}\text { Taxithelium planum (Brid.) } \\
\text { Mitt. }\end{array}$ & Co, Ter, Cas e Ep & $\begin{array}{c}\text { AM, PA, RO, RR, AL, BA, PE, DF, GO, MT, } \\
\text { MG, RJ, SP. }\end{array}$ & $\begin{array}{l}\text { Medeiros, D.L. \& Conceição, } \\
\text { G.M.217 (HABIT691) }\end{array}$ \\
\hline
\end{tabular}


Quadro 1. Cont.

\begin{tabular}{|c|c|c|c|}
\hline Divisão/ Famílias / Espécies & Substratos & Distribuição Geográfica no Brasil & Voucher \\
\hline Sematophyllum subsimplex (Hedw.) Mitt. & $\mathrm{Co}$ & $\begin{array}{c}\text { AC, AM, AP, PA, RO, RR, TO, AL, BA, CE, } \\
\text { MA, PE, PI, SE, DF, GO, MS, MT, ES, MG, } \\
\text { RJ, SP, PR, RS, SC. }\end{array}$ & $\begin{array}{l}\text { Medeiros, D.L. \& Conceição, } \\
\text { G.M. } 241 \text { (HABIT734) }\end{array}$ \\
\hline \multicolumn{4}{|l|}{ Stereophyllaceae } \\
\hline $\begin{array}{l}\text { Entodontopsis leucostega (Brid.) W.R. } \\
\text { Buck \& Ireland } \\
\text { Splachnobryaceae }\end{array}$ & Co e Ter & $\begin{array}{l}\mathrm{AC}, \mathrm{AM}, \mathrm{PA}, \mathrm{RO}, \mathrm{RR}, \mathrm{TO}, \mathrm{BA}, \mathrm{CE}, \mathrm{MA}, \mathrm{PB}, \\
\quad \text { PE, PI, DF, GO, MS, MT, MG, RJ, SP. }\end{array}$ & $\begin{array}{l}\text { Medeiros, D.L. \& Conceição, } \\
\text { G.M.264 (HABIT705) }\end{array}$ \\
\hline Splachnobryum obtusum (Brid.) Müll. Hal. & Ter & AC, AM, AP, AL, CE, GO, MS, SP, RS. & $\begin{array}{c}\text { Medeiros, D.L. \& } \\
\text { Conceição,G.M. } 270 \\
\text { HABIT698) }\end{array}$ \\
\hline \multicolumn{4}{|l|}{ Marchantiophyta } \\
\hline $\begin{array}{l}\text { Cylindrocolea rhizantha (Mont.) R.M. } \\
\text { Schust.* }\end{array}$ & Ter & AC, BA, PE, GO, ES, RJ, SP. & $\begin{array}{l}\text { Medeiros, D.L. \& Conceição, } \\
\text { G.M.380 (HABIT806) }\end{array}$ \\
\hline \multicolumn{4}{|l|}{ Frullaniaceae } \\
\hline Frullania platycalyx Herzog* & $\mathrm{Co}$ & PA, RJ, PR, RS, SC. & $\begin{array}{l}\text { Medeiros, D.L. \& Conceição, } \\
\text { G.M.340 (HABIT812) }\end{array}$ \\
\hline \multicolumn{4}{|l|}{ Lejeuneaceae } \\
\hline Archilejeunea parviflora (Nees) Stephani & $\mathrm{Co}$ & $\begin{array}{c}\mathrm{AC}, \mathrm{AM}, \mathrm{AP}, \mathrm{PA}, \mathrm{RO}, \mathrm{RR}, \mathrm{AL}, \mathrm{BA}, \mathrm{PE}, \mathrm{MS}, \\
\text { MT, ES, MG, RJ, SP, RS, SC. }\end{array}$ & $\begin{array}{l}\text { Medeiros, D.L. \& Conceição, } \\
\text { G.M.248 (HABIT1067) }\end{array}$ \\
\hline $\begin{array}{l}\text { Lejeunea caulicalyx (Steph.) Reiner, M. E. } \\
\text { \& Goda }\end{array}$ & Co, Ter, Cas e Ep & $\begin{array}{c}\mathrm{AC}, \mathrm{PA}, \mathrm{RR}, \mathrm{AL}, \mathrm{BA}, \mathrm{PE}, \mathrm{MS}, \mathrm{MT}, \mathrm{ES}, \mathrm{RJ}, \\
\text { SP, PR. }\end{array}$ & $\begin{array}{l}\text { Medeiros, D.L. \& Conceição, } \\
\text { G.M.218 (HABIT712) }\end{array}$ \\
\hline $\begin{array}{l}\text { Acrolejeunea torulosa (Lehm. \& Lindenb.) } \\
\text { Schiffn }\end{array}$ & $\mathrm{Co}$ & $\begin{array}{l}\text { AC, AM, PA, RO, RR, AL, BA, MA, PE, } \\
\text { GO, MS, MT, ES, MG, RJ, SP, PR, RS. }\end{array}$ & $\begin{array}{l}\text { Medeiros, D.L. \& Conceição, } \\
\text { G.M.385 (HABIT718) }\end{array}$ \\
\hline $\begin{array}{l}\text { Cololejeunea minutissima (G.L. Smith) } \\
\text { Schiffn. }\end{array}$ & Ep & $\begin{array}{c}\text { AC, AM, RR, BA, PE, MS, MT, ES, MG, RJ, } \\
\text { SP, PR, SC. }\end{array}$ & $\begin{array}{l}\text { Medeiros, D.L. \& Conceição, } \\
\text { G.M.146 (HABIT886) }\end{array}$ \\
\hline \multicolumn{4}{|l|}{ Lepidoziaceae } \\
\hline Telaranea nematodes (Austin) M. Howe* & $\mathrm{Co}$ & $\begin{array}{c}\mathrm{AC}, \mathrm{AM}, \mathrm{RR}, \mathrm{BA}, \mathrm{CE}, \mathrm{SE}, \mathrm{DF}, \mathrm{GO}, \mathrm{MS}, \mathrm{MT}, \\
\text { ES, MG, RJ, SP, RS, SC. }\end{array}$ & $\begin{array}{l}\text { Medeiros, D.L. \& Conceição, } \\
\text { G.M.254 (HABIT701) }\end{array}$ \\
\hline \multicolumn{4}{|l|}{ Ricciaceae } \\
\hline Riccia vitalii Jovet-Ast & Ter & $\begin{array}{l}\text { AM, TO, AL, BA, CE, MA, PB, PE, PI, RN, } \\
\text { SE, GO, MS, ES, RS. }\end{array}$ & $\begin{array}{l}\text { Medeiros, D.L. \& Conceição, } \\
\text { G.M. } 322 \text { (HABIT900) }\end{array}$ \\
\hline R. weinionis Steph.* & Ter e Rup & $\begin{array}{c}\mathrm{BA}, \mathrm{CE}, \mathrm{PB}, \mathrm{SE}, \mathrm{GO}, \mathrm{MS}, \mathrm{MT}, \mathrm{ES}, \mathrm{MG}, \mathrm{RJ} \\
\text { SP, PR. }\end{array}$ & $\begin{array}{l}\text { Medeiros, D.L. \& Conceição, } \\
\text { G.M.230 (HABIT743) }\end{array}$ \\
\hline R. plano-biconvexa Steph. & Ep e Ter & $\begin{array}{c}\text { TO, AL, BA, CE, PE, RN, DF, GO, MT, ES, } \\
\text { RJ, PR, RS, SC. }\end{array}$ & $\begin{array}{l}\text { Medeiros, D.L. \& Conceição, } \\
\text { G.M.306 (HABIT721) }\end{array}$ \\
\hline
\end{tabular}

\section{RESULTADOS E DISCUSSÃO}

Foram registradas 34 espécies (Quadro 1), sendo 24 pertencentes à Bryophyta, distribuídas em 16 gêneros e 11 famílias; e 10 espécies a Marchantiophyta, distribuídas em oito gêneros e cinco famílias. Áreas com formações vegetacionais abertas apresentam maior riqueza de musgos (Silva \& Pôrto 2013; Silva et al. 2014a; Silva et al. 2014b), o que justifica a considerável ocorrência de representantes da divisão Bryophyta em relação à Marchantiophyta.

Das espécies listadas para a área de estudo, oito são novos registros para o estado do Maranhão, Riccia weinionis Steph., Telarenea nematodes (Gottsche ex Austin) M.A.Howe, Cylindrocolea rhizantha (Mont.) R.M. Schust., Calymperes pallidum Mitt., Fissidens serratus Müll. Hal., Isopterygium affusum Mitt. e Barbula indica (Hook.) Spreng. (Quadro 1). Barbula arcuata Giff., além de ser novo registro para o Maranhão, é também a primeira citação para a região Nordeste do Brasil.

Além de Octoblepharum albidum Hedw. (Calymperaceae), comum para a região devido à alta disponibilidade de palmeiras, também por serem comuns em ambientes ensolarados (Floreschütz 1964), incluindo ainda, os antropizados (Yano 1992), as espécies mais frequentes em número de amostras foram Taxithelium planum (Brid.) Mitt. (36 ocorrências), espécie corriqueira e com ampla distribuição para o Neotrópico (Gradstein et al. 2001), e Fissidens angustelimbatus Mitt. (17 ocorrências), que segundo Florschütz (1964), é comum em barrancos de riachos.

O levantamento de briófitas no Povoado Buriti Corrente foi relativamente significativo, levando em consideração o número total de espécies que o Estado apresenta atualmente, o que equivale a $38 \%$ do total das espécies registradas para o estado do Maranhão. 


\section{AGRADECIMENTOS}

À Universidade Estadual do Maranhão, ao Centro de Estudos Superiores de Caxias pelo apoio e infraestrutura disponibilizada para a pesquisa. À Fundação de Amparo à Pesquisa e ao Desenvolvimento Científico e Tecnológico do Maranhão pelo apoio financeiro através edital FAPEMA ${ }^{\circ}$ 026/2013 CBIOMA/Apoio ao Programa Acervos do Maranhão Coleções Biológicas e Arqueológicas. Ao Pesquisador Denilson Fernandes Peralta, do Instituto de Botânica de São Paulo, pelo auxílio prestado nas identificações.

\section{REFERÊNCIAS}

Batista, W. V. S. M., Pôrto, K.C. \& Santos, N. D. 2018. Distribution, ecology, and reproduction of bryophytes in a humid enclave in the semiarid region of northeastern Brazil. Acta Botanica Brasilica 32(2): 303-313.

Buck, W. R. \& Goffinet, B. 2000. Morphology and classification of mosses. In Bryophyte Biology (A. J. Shaw \& B. Goffinet, eds.). Cambridge University Press, Cambridge, p.71-123.

Buck, W. R. 2003. Guide to the plants of Central French Guiana. Part 3. Mosses. Memoirs of The New York Botanical Garden, (76):1-167.

Costa, D. P. \& Peralta, D. F. 2015. Bryophytes diversity in Brazil. Rodriguésia 66(4): 1063-1071.

Crandall-Stotler, B. J., Stotler, R. E. \& Long, D. G. (2008 ['2009’]). Morphology and classification of the Marchantiophyta. In Bryophyte Biology (B. Goffinet, A. J. \& Shaw, eds.). New York: Cambridge University Press. p. 1-54.

Flora Do Brasil 2020 em construção. Jardim Botânico do Rio de Janeiro. Disponível em: http://floradobrasil.jbrj.gov.br/jabot/listaBrasil/ PrincipalUC/PrincipalUC.do. Acesso em 09.07. 2015.

Florschütz, P.A. 1964. The Mosses of Suriname. Part. I. In Flora of Suriname (J. Lanjouw ed.). E. J. Brill, Leiden, p. 1-271.

Glime, J.M. 2013. Bryophyta: Bryopsida In Bryophyte Ecology. Physiological Ecology. Ebook sponsored by Michigan Technological University, International. Association of Bryologists, p 1-24. Disponível em: < http://digitalcommons.mtu.edu/bryophyteecology1/13/>. Acesso em 10.06.2018.
Gradstein, S. R., Churchill, S. P. \& Salazar-Allen, N. 2001. Guide to the Bryophytes to Tropical America. Memoirs of The New York Botanical Garden, (86):1-577.

Gradstein, S.R. \& Costa, D.P. 2003. The Hepaticae and Anthocerotae of Brazil. Memoirs of The New York Botanical Garden, (87) 1-318.

Goffinet, B. \& Shaw, J. 2009. Bryophyte Biology, Cambridge University Press, New York, U.S.A. 581p.

Peralta, D. F., Brito, E. S., Varão, L. F., Conceição, G. M. \& CUNHA, I. P. R. 2011. Novas Ocorrências e Lista de Briófitas do Estado do Maranhão, Brasil. Pesquisa em foco 19 (1):63-78.

Proctor, M. C. F., Oliver, M.J., Wood, A.J., Alpert, P., Stark, L., Cleavitt, N. \& Mishler, B. 2007. Desiccation-tolerance in bryophytes : a review Desiccation-tolerance in bryophytes : a review. The American Bryological and Lichenological Society 110 (2): 595-621

Renzaglia, K. S., Villareal, J. C. \& Duff, R. J. 2009. New insights into morphology, anatomy, and systematic of hornworts. In Bryophyte Biology (B. Goffinet \& A. J. Shaw, eds.). Cambridge University Press, New York, p. 138-171.

Sharp, A.J., Crum, H. \& Eckel, P.M. 1994. The moss flora of Mexico. Memoirs of The New York Botanical Garden, 69(1-2):1-1113.

Schuster, R.M. 1980. The Hepaticae and Anthocerotae of North America. Columbia University Press, New York. 1334p.

Silva, M. \& Pôrto, K.C. 2013. Bryophyte communities along horizontal and vertical gradients in a human-modified atlantic forest remnant. Canadian Journal of Zoology 91 (3):155 -166.

Silva, T. O., Silva, M. P. P. \& Pôrto, K. C. 2014a. Briófitas de Afloramentos Rochosos do Estado de Pernambuco, Brasil. Boletim do Museu de Biologia Mello Leitão (Nova Série) 36:85-100.

Silva, J., Santos, N.D. \& Pôrto, K.C. 2014b. Beta-diversity: effect of geographical distance and environmental gradients on the rocky outcrop bryophytes. Cryptogamie, Bryologie 35(2):133 -163.

Yano, O. 1992. Novas localidades de musgos nos estados do Brasil. Acta Amazonica, 22(2): 197-218.

Yano, O., Juçara Bordin, J. \& Peralta, D.F. 2009. Briófitas dos estados do Ceará, Maranhão, Paraíba, Piauí e Rio Grande do Norte (Brasil). Hoehnea 36(3): 387-415.

Vieira, H, C., Oliveira, R, R., Silva, M. L., Silva, D. L. S. \& Conceição, G.M. 2017. Brioflora do estado do Maranhão, Brasil. Acta Brasiliensis, 1(2): 8-12.

Zander, R. H. 1993. Genera of the Pottiaceae: mosses of harsh environments. Bulletin of the Buffalo Society of Natural Sciences, (32): 1-378. 